\title{
Black holes with unusual topology
}

\author{
L. Vanzo \\ Dipartimento di Fisica, Università di Trento \\ and Istituto Nazionale di Fisica Nucleare, \\ Gruppo Collegato di Trento, Italia
}

may 1997

\begin{abstract}
The Einstein's equations with a negative cosmological constant admit solutions which are asymptotically anti-de Sitter space. Matter fields in anti-de Sitter space can be in stable equilibrium even if the potential energy is unbounded from below, violating the weak energy condition. Hence there is no fundamental reason that black hole's horizons should have spherical topology. In anti-de Sitter space the Einstein's equations admit black hole solutions where the horizon can be a Riemann surface with genus $g$. The case $g=0$ is the asymptotically anti-de Sitter black hole first studied by Hawking-Page, which has spherical topology. The genus one black hole has a new free parameter entering the metric, the conformal class to which the torus belongs. The genus $g>1$ black hole has no other free parameters apart from the mass and the charge. All such black holes exhibits a natural temperature which is identified as the period of the Euclidean continuation and there is a mass formula connecting the mass with the surface gravity and the horizon area of the black hole. The Euclidean action and entropy are computed and used to argue that the mass spectrum of states is positive definite.
\end{abstract}

PACS numbers: $\quad 04.20 .-q, 04.70 . B w, 04.70 . D y$

*e-mail:vanzo@science.unitn.it 


\section{Introduction}

In general relativity it was widely believed that black holes formed by gravitational collapse should have spherical horizon [1]. In the stationary case this is ensured by Hawking's theorem [2], under the assumption of asymptotic flatness and positivity of matter energy. The "topological censorship theorem" of Friedmann, Schleich and Witt [3] is another indication of the impossibility of non spherical horizons. The theorem states that in a globally hyperbolic, asymptotically flat spacetime, any two causal curves extending from past to future null infinity are homotopic. As pointed out by Jacobson and Venkataramani 画, a black hole with toroidal surface topology would provides a possible violation of topological censorship, as a light ray from past infinity linking with the hole of the torus and then back to future infinity would not be deformable to a light ray traveling from past to future outside the black hole. Thus the hole must quickly close up, before a light ray can pass through. In fact, as was shown by Shapiro, Teutolsky and Winicour [5], a temporarily toroidal horizon can form in gravitational collapse, in a way consistent with the theorems. For non stationary black holes, and under the assumptions of asymptotic flatness and the dominant energy condition for matter fields, Gannon [6] proved that a smooth black hole must be either a two-sphere or a torus. All these results made essential use of the condition of asymptotic flatness, which entails a vanishing cosmological constant.

The Einstein's equations with cosmological term, $\Lambda$, admit black hole solutions which are asymptotic to either de Sitter $(\Lambda>0)$ or anti-de Sitter $(\Lambda<0)$ space. These solutions have spherical horizon and obey thermodynamics laws like asymptotically flat black holes [7] 9]. In de Sitter space, one can find locally static solutions of the form

$$
d s^{2}=-V d t^{2}+V^{-1} d r^{2}+r^{2} d \sigma^{2} \quad V=C-\frac{2 m}{r}-\frac{\Lambda r^{2}}{3}
$$

for any $C$, provided the two dimensional line element $d \sigma^{2}$ has constant curvature $k=2 C$. Then for $C>0$ we have the asymptotically de Sitter black hole, with positive mass and spherical horizons. If $C<0$, the black hole interpretation of the solution is lost unless the mass parameter is negative. In anti-de Sitter space the situation is just the opposite. In $2+1$-dimensions there are the recently discovered locally anti-de Sitter black hole solutions [10], which have constant curvature everywhere not just asymptotically at infinity, and the Brill's multi-black hole's solution [11]. The horizon of a 2+1-dimensional spacetime is a closed line, which leaves not much space for introducing non trivial topology. On the other hand, there do not seem to exist a reasonable, higher dimensional generalization of the BTZ's black hole. The metrics recently found have horizons with spherical topology, but all the anti-de Sitter conserved charges are infinite [12]. So apparently, one had to give up the condition of constant curvature. Planar and cylindrical black holes in anti-de Sitter space were indeed discovered by Lemos [13,,14], which upon compactification became toroidal. Open and closed black strings [15] are also likely to form topologically toroidal black holes [16]. On the other hand, Aminneborg et al. [17] presented a class of solutions in 3+1-dimensions, displaying the causal structure characteristic of black holes, and having constant negative curvature everywhere. Hence they was locally isometric to anti-de Sitter space but, surprisingly, showed an event horizon with the topology of a Riemann surface with arbitrary genus. Finally, R. Mann [18], and then D. Brill in collaboration with J. Louko [11], introduced a class of black solutions admitting all the above horizon topologies, which can have both positive or negative mass, which can be charged, and which have a curvature singularity in the origin'. At the moment it was unclear whether these topological black holes could result from gravitational collapse but, since then, this question was also settled affirmatively [20]. So we finally have topologically non trivial black holes, albeit in anti-de Sitter space.

\footnotetext{
${ }^{1}$ Recently, the author also met the uncharged version of Mann' solution 19.
} 
Although anti-de Sitter space does not seem to correspond to the world in which we live, its importance has been noticed in many occasions [21 26]. Two features seem worth mentioning. Firstly, anti-de Sitter and Weyl conformal gravity are the only type of gravity which have a consistent interaction with massless higher spin fields [27] and, secondly, consistent anti-de Sitter strings exist for any $D \neq 26$ (or $D \neq 10$ ) [28], provided the cosmological term has the critical value which is required by anomaly cancellation.

In this paper we would like to investigate the thermodynamics properties of the topological black holes from the point of view of the Euclidean formulation (for a detailed treatment of the Hamiltonian thermodynamics of asymptotically anti-de Sitter black holes see [29]). We point out that higher genus black holes are really "cosmological black holes", inasmuch as their size is the size of the (anti-de Sitter) universe itself. Hence they could only exist during the inflationary era, when the cosmological constant was not small. The toroidal black hole, on the other hand, can exist in a virtually flat space, as the size is governed by the mass and the conformal class of the torus, rather than by the cosmological constant.

In Sec. (I), we begin by presenting the metric and discussing the relevant geometric features, including the asymptotic behaviour at infinity. We shall not discuss entirely the causal structure (it is presented in [30]), nor we make it confident how the black hole could result from gravitational collapse of some, topologically non trivial (i.e. non spherical) matter configuration (this is explained in [20]). In Sec. (II), we define the mass and show it obeys a Smarr-like formula. We point out that, due to the asymptotic behaviour of the metric, there is no way to make finite the Hamiltonian than subtracting a reference background in the same topology class of the actual solution. The natural choice would seem to be the solutions of Åminneborg et al., to which the black hole approaches asymptotically, but a thermodynamics argument will favor a rather different choice. In Sec. (III), we determine the off-shell Euclidean action and use it to evaluate the entropy of the black hole. Some discrepancies regarding the mass spectrum will then be resolved.

In the following, we shall use the curvature conventions of Hawking-Ellis's book [1] and employ Planck dimensionless units.

\subsection{The topological black holes}

The class of metrics to consider is

$$
d s^{2}=-V d t^{2}+V^{-1} d r^{2}+r^{2} \sigma_{i j} d x^{i} d x^{j}
$$

where $\sigma_{i j}$ is the metric of a two-manifold, $S$, which is not assumed to be a topological sphere, and $V=f(r)$. The non vanishing components of the Ricci tensor are

$$
R_{t t}=-V^{2} R_{r r}=\frac{1}{2} V V^{\prime \prime}+\frac{V V^{\prime}}{r} \quad R_{i j}=\mathcal{R}_{i j}-\left(r V^{\prime}+V\right) \sigma_{i j}
$$

where the calligraphic's $\mathcal{R}_{i j}$ refers to $\sigma_{i j}$. Now one verifies immediately that the function

$$
V=\kappa-\frac{k^{\prime}}{r}+\frac{r^{2}}{\ell^{2}}
$$

makes the metric to satisfy Einstein's equations with negative cosmological constant, $R_{a b}=\Lambda g_{a b}$, $\Lambda=-3 \ell^{-2}$, for any pair $\left(\kappa, k^{\prime}\right)$. The surprising fact is that for this to be true, the two dimensional metric $\sigma_{i j}$ must satisfy the equations for a constant curvature surface, which need not be a sphere, namely $\mathcal{R}_{i j}=\kappa \sigma_{i j}$ and $\mathcal{R}=2 \kappa$. Therefore if $\kappa=-q^{2}<0$, the two-manifold $S$ must be a surface with constant, negative curvature. If this surface is compact and orientable, then it must be a Riemann surface of genus $g>1$ for $q^{2}>0$. If $q=0$, then the surface is a torus, and $q= \pm i / R$ gives a sphere of radius $R$. Actually, the parameter $q$ is fictitious as long as non zero, since we 
can always rescale $t, r, k^{\prime}$ and $\sigma_{i j}$ so as to achieve that $q=1$. Hence we take the metric of the uncharged, genus- $g$, black hole in the form

$$
d s^{2}=-\left(-1-\frac{2 \eta}{r}+\frac{r^{2}}{\ell^{2}}\right) d t^{2}+\left(-1-\frac{2 \eta}{r}+\frac{r^{2}}{\ell^{2}}\right)^{-1} d r^{2}+r^{2} \sigma_{i j} d x^{i} d x^{j}
$$

where now $\mathcal{R}_{i j}=-\sigma_{i j}$ describes a Riemann surface with genus $g>1$ and Euler number $\chi_{g}=2-2 g$. In the genus one case, we pick a complex number $\tau$, with $\operatorname{Im} \tau>0$ (this is known as the Teichmüller complex parameter of the torus). Such a complex number specify a class of conformally equivalent tori, two tori being equivalent if and only if the respectives Teichmüller parameters are connected by a fractional linear transformation with integer coefficients. We shall write the flat metric of the torus in the form

$$
d \sigma^{2}=\sigma_{i j} d x^{i} d x^{j}=|\tau|^{2} d x^{2}+d y^{2}+2 \operatorname{Re} \tau d x d y
$$

where the pair $(x, y)$ ranges over the closed unit square in $\mathbb{R}^{2}$. The toroidal, uncharged black hole metric is now

$$
d s^{2}=-\left(-\frac{2 \eta}{r}+\frac{r^{2}}{\ell^{2}}\right) d t^{2}+\left(-\frac{2 \eta}{r}+\frac{r^{2}}{\ell^{2}}\right)^{-1} d r^{2}+r^{2}\left(|\tau|^{2} d x^{2}+d y^{2}+2 \operatorname{Re} \tau d x d y\right)
$$

Let $\delta(a, b)=1$ for $a=b$ and zero otherwise. From the Gauss-Bonnet theorem, the area of $S$ is

$$
\mathcal{A}=-2 \pi \chi_{g}+|\operatorname{Im} \tau| \delta(g, 1)=4 \pi(g-1)+|\operatorname{Im} \tau| \delta(g, 1)
$$

The metric possesses an irremovable singularity at $r=0$, because the invariant $R_{a b c d} R^{a b c d}$ blows up like $r^{-6}$ near $r=0$. Therefore, in the following, we shall study the metric for $r>0$ only.

We consider now whether the space represents a genuine black hole. The standard procedure to analyze black holes is to investigate the causal structure. In the $g>1$ case, the lapse function of the metric (1.4) always has a real root at some $r_{+}$. This is the solution of the cubic equation $r^{3}-\ell^{2} r-2 \eta \ell^{2}=0$, and the character of the roots depends on the sign of the discriminant, $\mathcal{D}=\eta^{2} \ell^{4}-\ell^{6} / 27$. If $\mathcal{D}>0$ and $\eta>0$ then

$$
r_{+}=\frac{2^{1 / 3} \ell^{2}}{3\left[2 \eta \ell^{2}+2(\mathcal{D})^{1 / 2}\right]^{1 / 3}}+\frac{\left[2 \eta \ell^{2}+2(\mathcal{D})^{1 / 2}\right]^{1 / 3}}{2^{1 / 3}}
$$

is the only real root, the singularity is spacelike and hidden inside an event horizon. If $\mathcal{D}>0$ but $\eta<0$ there is one negative real root, the lapse function is positive in the range $r>0$, and $r=0$ is a naked singularity. If $\mathcal{D}<0$, the allowed range for $\eta$ is $-\ell / 3 \sqrt{3} \leq \eta \leq \ell / 3 \sqrt{3}$. If $\eta>0$, there is one positive root which can be written as

$$
r_{+}=\frac{2 \ell}{\sqrt{3}} \cos (\theta / 3) \quad \cos \theta=\frac{3 \sqrt{3} \eta}{\ell}
$$

where $\theta \in[0, \pi / 2]$, the other two roots being real and negatives. Again the singularity is spacelike and hidden. If $\eta<0$ there are two positive roots, $r_{+}$and $r_{-}$, with $r_{+}>r_{-}$, corresponding to the choices $\theta / 3$ and $(\theta+4 \pi) / 3$ in Eq. (1.9), given $\theta \in[\pi / 2, \pi]$, and one negative root. Again the greater root represents an event horizon, and the region in between the two positive roots resembles the Reissner-Nordström solution. In the region $0<r<r_{-}$the lapse function is positive, so the singularity is timelike and $r=r_{-}$represents an inner Cauchy horizon. The structure of this black hole is then quite complex. As we shall see, $\eta$ is related to the mass of the black hole, hence what we have here is a putative, negative mass black hole with an acceptable causal structure, the allowed range of "negative mass" being $M>-\ell / 3 \sqrt{3}$. At last, $\eta=-\ell / 3 \sqrt{3}$ gives a naked singularity and the solution has no black hole interpretation. It corresponds to the extreme limit where the inner horizon has the same location as the outer horizon, $r_{-}=r_{+}$, and 
it will play an important role when developing the thermodynamics Euclidean theory. Finally, there is a case whereby $\mathcal{D}=0$, or $\ell^{2}=27 \eta^{2}$, for which again there is only one positive root at $r_{+}=6 \eta$, the other two being equal but negatives. The genus one case is simpler, as the only positive root is at $r_{+}=\left(2 \eta \ell^{2}\right)^{1 / 3}$.

In all cases, the root $r_{+}$makes the hypersurfaces $r=r_{+}$an event horizon. The metric admits a Kruskal like extension in which the $r=0$ singularity is spacelike (as in the Schwarzschild solution), the reason being that the lapse function changes sign by crossing the horizon, except when $\eta<0$, in which case the singularity is timelike. Because of this fact, each future directed null geodesic behind the horizon will inevitably crash into the singularity at $r=0$, so it can never reach infinity. A related fact is that the expansion of each $r=$ constant surface, with $r<r_{+}$, is negative and as such it is a closed trapped surface. The solution therefore represents a black hole for all $\eta>-\ell / 3 \sqrt{3}$, for $g>1$, or for all positive $\eta$ if $g=1$. The horizon has a portion to the future of the static region $r>r_{+}$, and a portion to the past. The two sheets intersect in a genus- $g$ Riemann surface, which is the fixed point set of the time translation symmetry of the solution. The horizon is thus a bifurcate Killing horizon. It has a surface gravity, $\kappa_{g}$ for the genus- $g$ case, which can be computed by standard means as

$$
\kappa_{g}=\frac{3 r_{+}^{2}-\ell^{2}}{2 r_{+} \ell^{2}} \quad \kappa_{1}=\frac{3 r_{+}}{2 \ell^{2}}
$$

The surface gravity is non negative and vanishes only for the extreme solution, when $\eta=-\ell / 3 \sqrt{3}$ and $r_{+}=\ell / \sqrt{3}$. The area section of the horizon is

$$
A=4 \pi r_{+}^{2}(g-1)+\delta(g, 1) r_{+}^{2}|\operatorname{Im} \tau|
$$

where $\tau$ is the Teichmüller parameter of the torus. The mass of the black hole is, unlike the geometry, a rather delicate matter, and we shall discuss this question after having analyzed few asymptotics property of the metric.

To understand the geometrical origin of the genus- $g$ surfaces, let us pause for a moment with the black hole and consider the solution with $\eta=0$ and $\kappa=-q^{2}$ not, normalized to -1 . The curvature tensor for this solution is $R_{a b c d}=-\ell^{-2}\left[g_{a c} g_{b d}-g_{a d} g_{b c}\right]$, which shows that the space is locally isometric to the universal covering of anti-de Sitter space. The surprise comes when computing the curvature tensor of the $r=$ constant surfaces. It is given by $\mathcal{R}_{i j k l}=$ $-q^{2}\left[\sigma_{i k} \sigma_{j l}-\sigma_{i l} \sigma_{j k}\right]$, and therefore it describes a space of constant, negative curvature again. Anti-de Sitter space, AdS for short, is the maximally symmetric space which is obtained by restricting the metric $d s^{2}=-d x^{2}-d v^{2}+d y^{2}+d z^{2}+d u^{2}$ in $\mathbb{R}^{5}$, with rectangular coordinates $(x, v, y, z, u)$, to the hyperboloid

$$
-x^{2}+y^{2}+z^{2}+u^{2}-v^{2}=-\ell^{2}
$$

The cosmological constant figuring in Einstein's equations is $\Lambda=-3 \ell^{-2}$. The topology of the space is that of $S^{1} \times \mathbb{R}^{3}$, but notice that each circle $x^{2}+v^{2}=\tau^{2}$ gives a closed timelike curve in AdS. Hence we pass to the covering by opening the circle into a real line. Given this, we note that by fixing $v^{2}-u^{2}=\ell^{2} \xi^{2}$ to be greater than $\ell^{2}$, i.e. $\xi^{2}>1$, makes the three remaining coordinates to range over hyperbolic two-space, which we denote by $H^{2}$. The orbits of constant $\xi$ describe uniformly accelerated observers in anti-de Sitter space, and we shall see now that the remaining $H^{2}$, which carries a positive definite metric, is the acceleration horizon of such observers. To this aim, we make use of the following parametrization of the hyperboloid

$$
\begin{aligned}
& x=\ell \sqrt{1+q^{-2} \xi^{2}} \cosh \rho \\
& y=\ell \sqrt{1+q^{-2} \xi^{2}} \sinh \rho \cos \theta \\
& z=\ell \sqrt{1+q^{-2} \xi^{2}} \sinh \rho \sin \theta
\end{aligned}
$$




$$
\begin{aligned}
& u=q^{-1} \ell \xi \cosh (q t / \ell) \\
& v=q^{-1} \ell \xi \sinh (q t / \ell)
\end{aligned}
$$

and then set $r^{2}=\ell^{2}\left(q^{2}+\xi^{2}\right)$. The induced metric takes the form

$$
d s^{2}=-\left(-q^{2}+\frac{r^{2}}{\ell^{2}}\right) d t^{2}+\left(-q^{2}+\frac{r^{2}}{\ell^{2}}\right)^{-1} d r^{2}+r^{2} d \sigma^{2}
$$

where $d \sigma^{2}=q^{-2}\left[d \rho^{2}+\sinh ^{2} \rho d \theta^{2}\right]$ is one of the many forms in which the metric of hyperbolic two-space $H^{2}$ is presented. Setting as before $q^{2}=1$, the metric differs from Eq. (1.4) by the absence of the crucial term $2 \eta / r$, but is otherwise identical.

The lapse function of the metric has a zero at $r_{+}=\ell$, which makes the metric of the three surface $r=r_{+}$degenerate. This surface is in fact a bifurcate event horizon, the future portion intersecting the past portion in a transverse $H^{2}$, which is the fixed point set ot the time translation symmetry. Although the metric displays the properties of a black hole, it is not in fact, as it represents the portion of AdS which is causally accessible to a family of accelerated observers. This is not the end of the story, as $H^{2}$ is non compact and we want a compact horizon. The $S O(2,3)$ symmetry group of AdS contains an $S O(1,2)$ subgroup acting on the $(x, y, z)$ sector of the five coordinates. This symmetry leaves unaffected the accelerated trajectories and only mixes the points in $H^{2}$, where it acts as a group of isometries. It is a well known fact that any Riemann surface with genus $g>1$ is the quotient space of $H^{2}$ by a discrete subgroup of isometries (roughly speaking, this is a subgroup whose elements can be labeled by an integer), acting in $H^{2}$ without fixed points (including infinity in $H^{2}$, so for example discrete translations are forbidden) and properly discontinuously (this means that the translates of any compact set are disjoints). Thus we may pick up such a discrete subgroup, say $\Gamma$, and make the quotient (i.e. the orbit space). This makes the horizon a compact Riemann surface of genus $g>1$. The genus one case apparently has not such interpretation, nevertheless it can also be obtained identifying points in AdS space [17] and the metric is Eq. (1.18) with $q^{2}=0$. As we shall see, thermodynamics arguments indicate that this solution has positive mass, even in the absence of the $2 \eta / r$ term in the metric. We shall call the resulting spacetime the RadS (Riemann-anti-de Sitter space), and we conclude that this is the asymptotic region of the topological black holes.

\subsection{The mass and size of the black holes}

As is well known, there is a certain amount of freedom in defining the mass of the black hole, as this involves the subtraction of a zero point of energy. Looking at the metric (1.18), it would seem natural to define the mass by taking RadS as a reference background, which has $\eta=0$, even if its topology is not that of anti-de Sitter space. However, for $g>1$ and for reasons to be explained below, we prefer to take as a reference background a metric in the class given by Eq. (1.4), with a "mass parameter" $\eta_{0}$. We shall also denote all quantities referring to the background with a subscript " 0 ". The two values of $\eta_{0}$ we will discuss are then $\eta_{0}=0$ and $\eta_{0}=-\ell / 3 \sqrt{3}$, which is the lowest possible value for the metric to admit a black hole interpretation, $\eta \leq \eta_{0}$ being a naked singularity. In the case $g=1$, the background will be the metric (1.6), but with $\eta_{0}=0$, which again is the lowest value for the metric to admit a black hole interpretation, $\eta_{0}<0$ being a naked singularity.

We shall now identify the mass of the black hole as the on-shell value of the Hamiltonian, with lapse function $N=\sqrt{V}$ and vanishing shift vector [29, 31] 33]. To this aim, one puts a timelike boundary at same large $r=R$ and uses the Hamiltonian of general relativity in a manifold with boundary, taking care of all the boundary terms. At the end, one takes the limit as $R$ goes to infinity. As the $t=$ constant slices are orthogonal to the timelike boundary at large 
distances which contains the Killing observers at "infinity", there are no "corner" terms in the Hamiltonian [34], and the mass is

$$
M=-\frac{1}{8 \pi} \int_{S_{g}} \sqrt{V}\left(\Theta-\Theta_{0}\right) r^{2} \sqrt{\sigma} d^{2} x
$$

where $S_{g}$ is a Riemann surface with genus $g, \Theta$ is the trace of the extrinsic curvature of $S_{g}$ as embedded in a $t=$ constant hypersurface, $\Theta_{0}$ is the same quantity as if $S_{g}$ were embedded in the reference spacetime, and the limit $R \rightarrow \infty$ is understood. The trace $\Theta$ can be computed as the covariant divergence of the normal vector field to the boundary at $r=R, \xi^{a}=\sqrt{V} \delta_{1}^{a}$, in the induced metric of the $t=$ constant slices , $h_{a b}=g_{a b}+u_{a} u_{b}$, where $u^{a}$ is the future pointing normal. It is

$$
\Theta=\frac{2}{R} \sqrt{-1-\frac{2 \eta}{R}+\frac{R^{2}}{\ell^{2}}} \simeq_{R \rightarrow \infty} \frac{2}{R} \sqrt{-1+\frac{R^{2}}{\ell^{2}}}+\frac{2 \eta}{R^{2}} \frac{1}{\sqrt{-1+\frac{R^{2}}{\ell^{2}}}}
$$

Similarly

$$
\Theta_{0} \simeq_{R \rightarrow \infty} \frac{2}{R} \sqrt{-1+\frac{R^{2}}{\ell^{2}}}+\frac{2 \eta_{0}}{R^{2}} \frac{1}{\sqrt{-1+\frac{R^{2}}{\ell^{2}}}}
$$

Therefore, asymptotically, $N\left(\Theta-\Theta_{0}\right) \simeq 2\left(\eta-\eta_{0}\right) R^{-2}$. One can repeat the calculation with the torus black hole metric, finding again the same result. From Eq. (1.19), in a condensed notation for any genus $g$, we obtain

$$
M=-\left(\eta-\eta_{0}\right) \chi_{g} / 2+\frac{\eta A}{4 \pi r_{+}^{2}} \delta(g, 1)=\left(\eta-\eta_{0}\right)(g-1)+\frac{\eta|\operatorname{Im} \tau|}{4 \pi} \delta(g, 1)
$$

We see that even if the "-1" in the lapse functions $N, N_{0}$ does not count asymptotically, the integration over the boundary must involve a Riemann surface in the background with the same genus of the actual solution. The topology of the background must then be asymptotically $\mathbb{R} \times$ $S_{g}$, with $S_{g}$ carrying a constant negative curvature metric (as required by Einstein's equations). This cannot be embedded in flat space (because then the curvature could not be negative everywhere), nor in the anti-de Sitter slices, which have topology $\mathbb{R} \times S^{2}$. If the background is to be a static solution of Einstein's equations, then presumably the metric (1.18) is the only one available which has no curvature singularities, and the metric (1.4) with $\eta_{0}=-\ell / 3 \sqrt{3}$ is the only one which has no black hole interpretation, although it has a naked singularity. The metric (1.4) makes sense even for $\eta<0$ [20,35, but again it has a curvature singularity in the origin. The $\eta_{0}=0$ background would seem preferable, as demanded by the topology of spacetime and by the absence of curvature singularities. We shall argue differently, however, when discussing the Euclidean theory.

In the following, it will be convenient to parametrize the reference background by $r_{0}$ rather than $\eta_{0}, r_{0}$ being the positive root of the cubic equation $r^{3}-\ell^{2} r-2 \eta_{0} \ell^{2}=0$. To the value $\eta_{0}=0$ corresponds then $r_{0}=\ell$ and to the critical value $\eta_{0}=-\ell / 3 \sqrt{3}$ corresponds the critical value $r_{0} \equiv r_{c}=\ell / \sqrt{3}$. This a double root of the lapse function, at which the background surface gravity vanishes. Finally, the toroidal background has $\eta_{0}=0$ and $r_{0}=0$.

If around the black hole there is a matter distribution with stress-energy tensor $T_{a b}$, then one can obtain a mass formula along the lines of [36], by integrating the Killing identity

$$
\nabla^{a} \nabla_{b} K_{a}=R_{b c} K^{c}
$$

for the Killing field $K^{a}$, over a spacelike hypersurface $\Sigma$, which is asymptotically orthogonal to the trajectories of the Killing observers at infinity and intersects the horizon in a two-surface 
$S_{g}$. To this we must subtract, in addition, the volume contribution of the background with the same genus (both the solution and the background have a divergent vacuum energy, because $\Lambda \neq 0$ ). This has an horizon at $r=r_{0}$ and a surface gravity, $\kappa_{0}$. The mass formula reads

$$
M=\frac{\kappa A}{4 \pi}-\frac{\kappa_{0} A_{0}}{4 \pi}+\frac{A}{4 \pi \ell^{2} r_{+}^{2}}\left(r_{0}^{3}-r_{+}^{3}\right)+\int_{\Sigma}\left(2 T_{a b}-T g_{a b}\right) K^{a} u^{b} d^{3} x
$$

the third term being the difference of the volume vacuum energy in the solution and the background. The mass so defined is also equal to the Abbott-Deser mass for asymptotically AdS spaces [26], if only one repeats their analysis in the present case, and satisfy the first law for any $g$, which in the vacuum reads

$$
d M=\frac{\kappa d A}{8 \pi}
$$

The knowledge of the mass allows one to obtain some result about the size of the topological black holes. The radius of the black hole as seen from the outside static region is the value, $r_{+}$, of the real positive root of the lapse. This depends on a single parameter, $\eta$, that we showed is related to the black hole mass. The scale of the solution is determined by the cosmological constant, or by $\ell$ (present estimates would put a value for $\ell$ not less than $10^{27} \div 10^{28} \mathrm{~cm}$, which is about the size of the observable universe). For $g>1$, according to Eq. (1.8), the black hole can have any size for masses grater than $\ell / \sqrt{27}$ and grows like $r_{+} \sim M^{1 / 3}$ for $M \ell \gg 1$. This seems to be the less interesting case for large values of $\ell$. The degenerate case $\mathcal{D}=0$, is a black hole with $r_{+}=6 \eta \sim M \sim \ell$, and the size of the black hole is the size of the universe. There is finally a case whereby $\mathcal{D}<0$. The mass of the black hole is bounded by a number of order $\ell$, the greater the mass the greater is the size, but this is always of order $\ell$. Hence there are no "small" topological, $g>1$ black holes, except for large values of the cosmological constant. In fact, the minimum size is $2 \ell / \sqrt{3}$ for $\mathcal{D} \geq 0$ and $\ell / \sqrt{3}$ if $\mathcal{D}<0$. If the black hole can radiate away its mass without changing the topology, then this would leave behind a cosmological horizon with finite size or a naked singularity. The toroidal black hole is more promising, as then $r_{+}=\left(2 \eta \ell^{2}\right)^{1 / 3}$. As a function of the mass this is

$$
r_{+}=\left(\frac{8 \pi \ell^{2} M}{|\operatorname{Im} \tau|}\right)^{1 / 3}
$$

and thus it depends on the conformal class of the torus. Now small black holes can exist with any mass and, within a given conformal class, they can exist for arbitrary large values of $\ell$. If the cosmological constant, though exceedingly small, is nevertheless finite, the toroidal black hole could exist in a virtually flat space.

\subsection{Euclidean formulation}

One approach to the thermodynamics of black holes, is to analyze the Euclidean action which one obtains under Wick rotation to imaginary time [7]. The Euclidean black hole solution is obtained by rotating the time coordinate to imaginary values, and exist as a Riemannian metric for $r>r_{+}$. In the Euclidean section of the metric, the imaginary time plays the role of an angular coordinate, where the rotation "axis" is just the horizon. Therefore the metric will have a conic singularity at $r=r_{+}$, unless the imaginary time is identified with the right periodicity, which is

$$
\beta_{+}=\frac{2 \pi}{\kappa}=[1-\delta(g, 1)] \frac{4 \pi \ell^{2} r_{+}}{3 r_{+}^{2}-\ell^{2}}+\delta(g, 1) \frac{4 \pi \ell^{2}}{3 r_{+}}
$$

An important exception to this is the critical solution with $\eta_{0}=-\ell / 3 \sqrt{3}$. This is the only solution for which the imaginary time can be identified with any period without loosing the 
regularity of the metric. This fact will have important consequences for the Euclidean theory. Unlike the asymptotically AdS black hole studied in [8,9, the period has no maximum value and is never zero, so the solution exist for any $\beta_{+}$. However, $\beta_{+}>2 \pi \ell$ corresponds to negative energy states, if the prescription $\eta_{0}=0$ is adopted. The temperature of the genus $g$ black holes is therefore, for $g>1$ and $g=1$ respectively

$$
T=\beta_{+}^{-1}=\frac{3 r_{+}^{2}-\ell^{2}}{4 \pi \ell^{2} r_{+}} \quad T=\frac{3 r_{+}}{4 \pi \ell^{2}}
$$

The quantum origin of this temperature is hidden here by our choice of units. The identification of the period in imaginary time (a classical concept) with the inverse temperature of the equilibrium state, has no classical analog since the required Wick rotation is really $t \rightarrow-i \hbar \beta$. That $T$ is a temperature can also be seen from the fact that one can construct the analog of the Hartle-Hawking quantum state as well as the analog of the Unruh state (work in preparation). To define the former, one imposes the boundary condition that the ingoing and outgoing fluxes of radiation, from and to timelike infinity, be equal. Both states have a temperature which is zero at infinity due to infinite redshift. However, zero rest mass particles escape to infinity arranged in a thermal flux with the temperature $T$, but their "angular distribution" is governed by the eigenfuctions of the Laplace operator on a Riemann surface rather than by the spherical harmonics.

The mass of the black hole as a function of the temperature is an important thermodynamics input. It can be obtained from the mass formula by expressing $r_{+}$in terms of $T$ using Eq. (1.28), which gives, for $g>1$ and $g=1$ respectively

$$
r_{+}=\frac{2 \pi \ell^{2} T}{3}\left[1+\sqrt{1+\frac{3}{4 \pi^{2} \ell^{2} T^{2}}}\right] \quad r_{+}=\frac{4 \pi \ell^{2} T}{3}
$$

For $g>1$, the mass is a rather complicated function of this temperature

$$
\begin{aligned}
M & =\frac{(g-1) 4 \pi^{3} \ell^{4} T^{3}}{27}\left(1+\sqrt{1+\frac{3}{4 \pi^{2} \ell^{2} T^{2}}}\right)\left[\left(2+\frac{3}{4 \pi^{2} \ell^{2} T^{2}}+2 \sqrt{1+\frac{3}{4 \pi^{2} \ell^{2} T^{2}}}\right)-\ell^{2}\right] \\
& -\eta_{0}(g-1) \geq-\left(\frac{\ell}{3 \sqrt{3}}+\eta_{0}\right)(g-1)
\end{aligned}
$$

This mass is an increasing function of $T$, with a large- $T$ behaviour $M \sim T^{3}$, in the full range $0 \leq T \leq \infty$ and the zero temperature state is a black hole with mass $M=-\left(\eta_{0}+\ell / 3 \sqrt{3}\right)(g-1)$. The mass then increases till the temperature reaches the value $T=1 / 2 \pi \ell$, at which the mass is $M=-\eta_{0}(g-1)$. The first prescription, $\eta_{0}=0$, gives then a massless black hole at finite temperature, at the end of a continuous spectrum of negative energy states, and the second prescription gives a continuous positive mass spectrum, although at this stage the terminology is conventional. However, it would seem natural to call "ground state" the state with zero temperature. For $g=1$ the mass is

$$
M=|\operatorname{Im} \tau| \frac{8 \pi^{2} \ell^{4}}{27} T^{3}
$$

Hence the stability condition, $\partial M / \partial T>0$, is fulfilled in every case. We shall now compute the off-shell Euclidean action of the black hole

$$
I=-\frac{1}{16 \pi} \int_{\mathcal{M}}(R-2 \Lambda) \sqrt{g} d^{4} x-\frac{1}{8 \pi} \int_{\partial \mathcal{M}} K \sqrt{h} d^{3} x
$$

where $\partial \mathcal{M}=S^{1} \times S_{g}$ is the boundary of the solution identified with period $\beta \neq \beta_{+}$at some fixed $r=R$, which will be taken to infinity at the end, and $K$ is the trace of the extrinsic curvature of 
the boundary. The Euclidean action so defined is a divergent function of the boundary location, and therefore it will be necessary to subtract from it the Euclidean action of a chosen background. For black holes which are asymptotically flat, de Sitter or anti-de Sitter, one can compute the difference of the Euclidean action of the actual solution with that of flat space, the four-sphere or the four-dimensional hyperbolic space respectively, these spaces being the Euclidean sections of the lorentzian metrics. In flat space, the Euclidean action comes entirely from the difference in the surface terms, the four-sphere has no boundary and the action is already finite without subtractions [7, 37], in hyperbolic four-space the surface integral of the solution cancel the surface integral of the background and the action comes again from the difference in four volumes [8].

In the present case, we have apparently no other choice than comparing the Euclidean action of the black hole with that of another solution in the same topological class (i.e same Euler number). This is because with no other topology will the metric cancel the divergences coming from the surface and volume terms in the action, without fine tuning the parameters. For example, choosing anti-de Sitter requires fine tuning of the cosmological constant of the background to achieve cancellation of the leading divergences. Another choice could be taking a background in the same topology class but with a metric chosen by hand to cancel divergences. In general, however, this will not be a solution of Euclidean Einstein's equations, the procedure appears a little bit arbitrary and, moreover, the mass was defined relative to a specific background.

Therefore we shall compute the difference between the Euclidean action of the black hole and that of a background in the same topology class, and for off-shell values of the inverse black hole's temperature. To agree with the mass definition, the $\eta_{0}$-parameter of the background will be either zero or $-\ell / 3 \sqrt{3}$. In doing so, one encounters a conical singularity in the solutions as well as the background, except in the second case. We notice that such singularity in the background would persist even for on-shell values of $\beta$, as the natural period of the background is different from $\beta_{+}$. To compute the effect of the conical singularity one cuts, out of the manifold, a small disk around the horizon (in the Euclidean black hole this is an axis of rotation) at $r=\varepsilon$, and then compute separately the action in the volume from $r=\varepsilon$ to $r=R$, and the disk. The contribution of the disk is given, as is well known [38 40], by the Gauss-Bonnet theorem and is

$$
\frac{1}{16 \pi} \int R \sqrt{g} d^{4} x=\frac{A}{4 \beta_{+}}\left(\beta_{+}-\beta\right)
$$

where $A$ is the area of the event horizon. The background contributes the same quantity or zero, depending on whether $r_{0}=\ell$, for the choice $\eta_{0}=0$, or $r_{0}=r_{c}=\ell / \sqrt{3}$ for the choice $\eta_{0}=-\ell / 3 \sqrt{3}$. In the former case, $A_{0}=4 \pi \ell^{2}(g-1)$ takes the place of $A$ and $\beta_{0}=2 \pi \ell$ the place of $\beta_{+}$. Finally, the conic contribution of the toroidal background vanishes, too. Since for both metrics $R=4 \Lambda$, the volume's difference in the action of the two metrics is

$$
\frac{\beta \mathcal{A}}{8 \pi \ell^{2}}\left(R^{3}-r_{+}^{3}+r_{0}^{3}-R_{0}^{3}\right)
$$

wher $\mathcal{A}=4 \pi(g-1)+\delta(g, 1)|\operatorname{Im} \tau|$ and $R_{0}$ is the radial coordinate of the boundary in the background metric. This must be matched to $R$ by requiring the two metrics to agree asymptotically, which gives (for the torus is $\eta_{0}=0$ )

$$
R_{0}=R-\frac{\left(\eta-\eta_{0}\right) \ell^{2}}{3 R^{2}}+O\left(R^{-3}\right)
$$

up to terms of higher order in $R^{-1}$. Finally there is the surface contribution, which involves the asymptotic of the extrinsic curvatures in the form (this is the integrand of the boundary term in Eq. (1.32), after subtraction)

$$
R^{2} N K-R_{0}^{2} N_{0} K_{0}=R^{2}\left(\frac{2 V}{R}+\frac{V^{\prime}}{2}\right)-R_{0}^{2}\left(\frac{2 V_{0}}{R_{0}}+\frac{V_{0}^{\prime}}{2}\right)
$$


where $N=\sqrt{V}$ and $N_{0}=\sqrt{V_{0}}$ are the lapse functions of the black hole and the background respectively. Using the matching condition one finds this to vanish at infinity, and therefore the surface term also vanishes. Finally, we eliminate $\eta$ in favor of the mass and we obtain the following off-shell Euclidean action, valid for any genus $g$ (we recall the Kronecker symbol $\delta(a, b)=1$ if $a=b$ and zero otherwise)

$$
I=\frac{A}{4 \beta_{+}}\left(\beta-\beta_{+}\right)+\left[1-\delta\left(r_{0}, r_{c}\right)\right] \frac{A_{0}}{4 \beta_{0}}\left(\beta_{0}-\beta\right)+\frac{\beta A\left(r_{0}^{3}-r_{+}^{3}\right)}{8 \pi \ell^{2} r_{+}^{2}}+\frac{\beta M}{2}
$$

Notice that the conic contribution of the background is absent if $r_{0}=r_{c}=\ell / \sqrt{3}$, which correspond to the zero temperature state, or what is considered a "negative mass" solution in [20,30]. Using the mass formula Eq. (1.24), one can write the action in the form

$$
I=\beta M-\frac{A}{4}+\left[1-\delta\left(r_{0}, r_{c}\right)\right] \frac{A_{0}}{4}
$$

From this formula it would seem that a mass shift, though moving the negative energy states to positive values, would leave unaffected the entropy. This is wrong, because shifting $M$ by $\eta_{0}=$ $-\ell / 3 \sqrt{3}$, removes at the same time the conic singularity in the background, and consequently affects the entropy. The on-shell action is $I$ evaluated at $\beta=\beta_{+}$, that is

$$
I=\left[1-\delta\left(r_{0}, r_{c}\right)\right] \frac{A_{0}}{4 \beta_{0}}\left(\beta_{0}-\beta_{+}\right)+\frac{\beta_{+} A\left(r_{0}^{3}-r_{+}^{3}\right)}{8 \pi \ell^{2} r_{+}^{2}}+\frac{\beta_{+} M}{2}
$$

As a function of the black hole's temperature and for $g>1$, this is

$$
\begin{aligned}
I & =\frac{M}{2 T}-\frac{4 \pi^{3} \ell^{4} T^{2}(g-1)}{27}\left[1+\sqrt{1+\frac{3}{4 \pi^{2} \ell^{2} T^{2}}}\right]^{3}+\frac{(g-1) r_{0}^{2}}{2 \ell}\left(\frac{r_{0}}{\ell}-\frac{2 \pi \ell}{\beta_{0}}\right) T^{-1} \\
& +\left[1-\delta\left(r_{0}, r_{c}\right)\right] \frac{A_{0}}{4}
\end{aligned}
$$

where $M$ is given by Eq. (1.30), and for the torus is

$$
I=-|\operatorname{Im} \tau| \frac{4 \pi^{2} \ell^{4} T^{2}}{27}
$$

The $T$ behaviour is exactly $-T^{2}$ for the torus and $-T^{2}$ asymptotically for higher genus, as for a massless boson gas in two spatial dimensions. In the tree approximation one identifies $I=-\log Z(\beta)$, the partition function of the black hole [37]. The density of states is the inverse Laplace transform of the partition function

$$
\rho(E)=\frac{1}{2 \pi i} \int_{\operatorname{Re} \beta=c} Z(\beta) e^{\beta E} d \beta
$$

For large energy the integral is dominated by the small- $\beta$ limit of the partition function, which is of order $\exp \left(-C \beta^{-2}\right), C>0$. Then the integrand has a saddle point at

$$
\beta \simeq(2 C / E)^{1 / 3}
$$

at which the second derivative of the logarithm of the integrand is positive. The path of steepest descent is then parallel to the imaginary axis and the integral gives

$$
\rho(E) \simeq \exp \left(C_{1} E^{2 / 3}\right) \quad C_{1}>0
$$

for some computable new constant $C_{1}$. For genus one the result is exact, but in every case the exponent is precisely $A / 4$. This growth of $\rho(E)$ with $E$ makes it evident the existence of the partition function from the point of view of the "sum over states". The stability of the 
canonical ensemble is proved in [30], using the method of the reduced action, and is indicated by the positivity of the specific heat.

Adopting $\log Z(\beta)=-I$ as the partition function, where $I$ is the off-shell Euclidean action, allows one to evaluate the expectation value of the energy in the canonical ensemble. This is

$$
<E>=-\partial_{\beta} \log Z=M
$$

as it was expected, and the entropy is

$$
S=\frac{A}{4}-\pi r_{0}^{2}(g-1)\left[1-\delta\left(r_{0}, r_{c}\right)\right]
$$

where $A=r_{+}^{2}|\operatorname{Im} \tau|$ in the $g=1$ case. One can also derive these results from the on-shell Euclidean action, if only one takes the derivative of Eq. (1.38) or Eq. (1.39) with respect to T.

\subsection{Discussion}

From Eqs. (1.43), (1.44) it is clear how to proceed. First we notice that the entropy is exactly one quarter the area of the event horizon in the genus one black hole, the Hamiltonian mass is always positive and equal to the mean energy in the canonical ensemble. This is satisfactory and we shall not discuss this case any more.

For the higher genus black holes, on the other hand, we have the choices $r_{0}=\ell \neq r_{c}$, which means the reference background is the $\eta=0$ solution of Åminneborg et al., or $r_{0}=r_{c}=\ell / \sqrt{3}$, which is the zero temperature state corresponding to a naked singularity. We shall now discuss these two cases in order. The former choice was used in [19, 30] to define what is meant by the mass of the black holes. We stress that the prescription by which one defines the Hamiltonian mass and the Euclidean action should be the same, otherwise one runs into inconsistencies and, moreover, one cannot compare the two (see [33] for a discussion of this relation between Hamiltonian mass and Euclidean action). Doing this consistently, we see from Eq. (1.30) that there is a continuum of negative energy states in the range $-\ell / 3 \sqrt{3} \leq M<0$ (with positive specific heat, nevertheless), and the entropy picks up a topological contribution in the higher genus black holes (the sign of this was mistakenly taken to be positive in [19]). The disaster is that the entropy becomes negative precisely when the temperature falls below the value $T_{0}=1 / 2 \pi \ell$, at which the black hole's mass crosses zero becoming negative. A negative entropy does not make sense, so either one removes by hand the negative mass spectrum or interpret the negative entropy as meaning that negative energy states have an exponentially small probability of order $\exp (-3 \pi(g-1) /|\Lambda|)$. However, these putative negative mass black holes are perfectly acceptable solutions resembling much the Reissner-Nordström solution, and have positive specific heat as the mass versus $T$ relation is concave upward everywhere. It seems rather arbitrary to cut them off, and we think, indeed, that this would be wrong.

The second choice assign zero mass to the critical solution with $\eta_{0}=-\ell / 3 \sqrt{3}$, or $r_{0}=r_{c}$, which is not in fact a black hole (see [30] for the causal Penrose diagram). Looking at the mass spectrum, Eq. (1.30), we see it is positive for $T>0$ and zero only at $T=0$. Notice that the

$\eta=0$ solution of Aminneborg et al. has now positive mass, equal to $\ell(g-1) / \sqrt{27}$, but no curvature singularity at all. The near-to-zero temperature solution is a near-to-extreme black hole, which becomes a naked singularity at $T=0$ (the extreme Reissner-Nordström solution, instead, is a black hole, but one on the verge of developing a naked singularity. It is only as a result of quantum emission that is driven away towards the non extreme solutions [41]). The third law of thermodynamics is thus perfectly consistent with the third law of black hole mechanics, according to which extreme solutions are forbidden. This is a satisfactory result, and we look now to the entropy of the black hole.

Making the choice $\eta_{0}=-\ell / 3 \sqrt{3}$, which is equivalent to $r_{0}=r_{c}$, the unwanted negative term in the entropy formula disappears, leaving a positive definite entropy equal to one-quarter 
the area of the event horizon, in agreement with [30] (which, however, have a partly negative mass spectrum since the $\eta=0$ solution was adjusted to zero mass). This indicates it is not $\eta$ that is related to the mass, but rather $\eta+\eta_{0}$ is. As discussed in the text, the reason for the disappearance of the unwanted term is quite subtle. The reference extreme background is the only solution, among the class considered, whose Euclidean section can be identified to any period in imaginary time without loosing the regularity of the metric. In all other backgrounds within the same topology class, there is a conic singularity even on-shell, which suddenly disappears in the extreme limit. We conclude that the topological black holes, at least in semiclassical quantum gravity, forms a well behaved sequence of positive mass solutions in anti-de Sitter gravity, with a stable thermodynamics. However, one cannot exclude the existence of topological transitions between different genus sectors. In this context, one may note that the genus of the black hole, or its conformal class in the toroidal case, is at this level a free, non dynamical parameter. This is unsatisfactory, and a better origin should be sought. As AdS is a possible ground state for string theory, it is not unlike that string theory and its parentage with the mathematics of the Riemann surfaces could do better than us.

\section{Acknowledgments}

The author acknowledge useful conversations with S. Zerbini, G. Cognola and R. Parentani.

\section{References}

[1] S.W. Hawking and G.F. Ellis. The large scale structure of spacetime. Cambridge University Press, Cambridge, England, (1973).

[2] S. W. Hawking. Commun. Math. Phys. 25, 152 (1972).

[3] J.L. Friedmann, K. Schleich and D.M. Witt. Phys. Rev. Lett. 71, 1486 (1993).

[4] T. Jacobson and S. Venkataramani. Class. and Quantum Grav. 12, 1055 (1995).

[5] S.L. Shapiro, S.A. Teutolsky and J. Winicour. Phys. Rev. D52, 6982 (1995).

[6] D. Gannon. Gen. Rel. and Grav. 7, 219 (1976).

[7] G.W. Gibbons and S. W. Hawking. Phys. Rev. D15, 2738 (1977).

[8] S. W. Hawking and D. N. Page. Commun. Math. Phys. 87, 577 (1983).

[9] J.D. Brown, J. Creighton and R. B. Mann. Phys. Rev. D50, 6394 (1994).

[10] M. Bañados, C. Teitelboim and J. Zanelli. Phys. Rev. Lett. 69, 1849 (1992).

[11] D. Brill. Helv. Phys. Acta. 69, 249 (1996).

[12] M. Bañados. gr-qc/9703040 (1997).

[13] J.P.S. Lemos. Class. and Quantum Grav. 12, 1081 (1995).

[14] J.P.S. Lemos. Phys. Lett. B 353, 46 (1996).

[15] G.T. Horowitz and A. Strominger. Nuc. Phys. B360, 197 (1991).

[16] J.P.S. Lemos and V.T. Zanchin. Phys. Rev. D54, 3840 (1996). 
[17] S. Åminneborg, I. Bengtsson, S. Holst and P. Peldan. Class. Quantum Grav. 13, 2707 (1996).

[18] R.B. Mann. Class. and Quantum Grav. 14, L109 (1997).

[19] L. Vanzo. gr-qc/9705004, (1997).

[20] R.B. Mann and W.L. Smith. gr-qc/9703007 (1997).

[21] C. Fronsdal. Rev. Mod. Phys. 37, 221 (1965).

[22] S.J. Avis, C.J. Isham and D. Storey. Phys. Rev. D18, 3565 (1978).

[23] S. Coleman and F. De Luccia. Phys. Rev. D21, 3305 (1980).

[24] P. Breitenlohner and D.Z. Freedman. Ann. Phys. 144, 249 (1982).

[25] F. Filthaut and C. Dullemond. Phys. Rev. D43, 485 (1991).

[26] L.F. Abbot and S. Deser. Nucl. Phys. B195, 76 (1982).

[27] E.S. Fradkin and M.A. Vasiliev. Ann. Phys. 177, 63 (1987).

[28] E.F. Fradkin and V.Ya. Linetsky. Phys. Lett. B261, 26 (1991).

[29] J. Louko and S.N. Winters-Hilt. Phys. Rev. D54, 2647 (1996).

[30] D.R. Brill, J. Louko and P. Peldan. gr-qc/9705012 (1997).

[31] T. Regge and C. Teitelboim. Ann. of Phys. 88, 286 (1974).

[32] J.D. Brown and J.W. York. Phys. Rev. D47, 1407 (1993).

[33] S.W. Hawking and G.T. Horowitz. Class. Quantum Grav. 13, 1487 (1996).

[34] G. Hayward. Phys. Rev. D47, 3275 (1993).

[35] R. Mann. gr-qc/9705007 (1997).

[36] J.M. Bardeen, B. Carter and S.W. Hawking. Commun. Math. Phys. 31, 161 (1973).

[37] G.W. Gibbons and S.W. Hawking. Phys. Rev. D15, 2752 (1977).

[38] C. Teitelboim. Phys. Rev. D51, 4315 (1995).

[39] L. Susskind and J. Uglum. Phys. Rev. D50, 2700 (1994).

[40] V.P. Frolov, D.V. Fursaev and A.I. Zelnikov. Phys. Rev. D54, 2711 (1996).

[41] L. Vanzo. Phys. Rev. D55, 2192 (1997). 